\title{
Front Matter: Volume 7452
}

, "Front Matter: Volume 7452," Proc. SPIE 7452, Earth Observing Systems XIV, 745201 (24 September 2009); doi: 10.1117/12.845513

SPIE Event: SPIE Optical Engineering + Applications, 2009, San Diego, California, SPIE. United States 


\section{PROCEEDINGS OF SPIE}

\section{Earth Observing Systems XIV}

James J. Butler

Xiaoxiong Xiong

Xingfa Gu

Editors

3-5 August 2009

San Diego, California, United States

Sponsored and Published by

SPIE

Volume 7452 
The papers included in this volume were part of the technical conference cited on the cover and title page. Papers were selected and subject to review by the editors and conference program committee. Some conference presentations may not be available for publication. The papers published in these proceedings reflect the work and thoughts of the authors and are published herein as submitted. The publisher is not responsible for the validity of the information or for any outcomes resulting from reliance thereon.

Please use the following format to cite material from this book:

Author(s), "Title of Paper," in Earth Observing Systems XIV, edited by James J. Butler, Xiaoxiong Xiong, Xingfa GU, Proceedings of SPIE Vol. 7452 (SPIE, Bellingham, WA, 2009) Article CID Number.

ISSN 0277-786X

ISBN 9780819477422

Published by

SPIE

P.O. Box 10, Bellingham, Washington 98227-0010 USA

Telephone +1 3606763290 (Pacific Time) · Fax +1 3606471445

SPIE.org

\section{Copyright (C) 2009, Society of Photo-Optical Instrumentation Engineers}

Copying of material in this book for internal or personal use, or for the internal or personal use of specific clients, beyond the fair use provisions granted by the U.S. Copyright Law is authorized by SPIE subject to payment of copying fees. The Transactional Reporting Service base fee for this volume is $\$ 18.00$ per article (or portion thereof), which should be paid directly to the Copyright Clearance Center (CCC), 222 Rosewood Drive, Danvers, MA 01923. Payment may also be made electronically through CCC Online at copyright.com. Other copying for republication, resale, advertising or promotion, or any form of systematic or multiple reproduction of any material in this book is prohibited except with permission in writing from the publisher. The CCC fee code is 0277-786X/09/\$18.00.

Printed in the United States of America.

Publication of record for individual papers is online in the SPIE Digital Library.

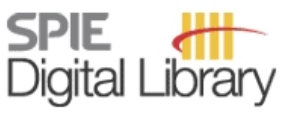

SPIEDigitalLibrary.org

Paper Numbering: Proceedings of SPIE follow an e-First publication model, with papers published first online and then in print and on CD-ROM. Papers are published as they are submitted and meet publication criteria. A unique, consistent, permanent citation identifier (CID) number is assigned to each article at the time of the first publication. Utilization of CIDs allows articles to be fully citable as soon they are published online, and connects the same identifier to all online, print, and electronic versions of the publication. SPIE uses a six-digit CID article numbering system in which:

- The first four digits correspond to the SPIE volume number.

- The last two digits indicate publication order within the volume using a Base 36 numbering system employing both numerals and letters. These two-number sets start with $00,01,02,03,04$, $05,06,07,08,09,0 A, 0 B \ldots 0 Z$, followed by 10-1Z, 20-2Z, etc.

The CID number appears on each page of the manuscript. The complete citation is used on the first page, and an abbreviated version on subsequent pages. Numbers in the index correspond to the last two digits of the six-digit CID number. 


\section{Contents}

ix Conference Committee

\section{SESSION 1 PRELAUNCH CALIBRATION}

745203 Spectral features: how to reduce them [7452-02]

H. van Brug, D. ten Bloemendal, B. de Goeij, TNO Science and Industry (Netherlands);

R. Vink, L. Maresi, ESA ESTEC (Netherlands)

745204 The extension of the NIST BRDF scale from $1100 \mathrm{~nm}$ to $2500 \mathrm{~nm}$ [7452-03]

H. W. Yoon, D. W. Allen, G. P. Eppeldaver, B. K. Tsai, National Institute of Standards and Technology (United States)

745205 Progress in BRDF calibration measurements in the SWIR [7452-04]

G. T. Georgiev, Sigma Space (United States); J. J. Butler, NASA Goddard Space Flight Ctr. (United States)

745206 Development of a filter radiometer monitor system for integrating sphere sources [7452-05] L. Ding, M. G. Kowalewski, J. W. Cooper, G. R. Smith, Science Systems and Applications, Inc. (United States); J. J. Butler, NASA Goddard Space Flight Ctr. (United States)

\section{SESSION 2 NPOESS PREPARATORY PROJECT}

745207 Radiometric calibration of the OMPS instruments for NPOESS [7452-06]

S. C. Bennett, Ball Aerospace \& Technologies Corp. (United States)

745208 Radiometric calibration of $100-\mathrm{cm}$ sphere integrating source for Visible/Infrared Imager/Radiometer Suite (VIIRS) [7452-07]

E. D. Kim, R. Menzel, J. R. Moore, Raytheon Space \& Airborne Systems (United States)

745209 VIIRS polarization testing [7452-08]

E. Waluschka, NASA Goddard Space Flight Ctr. (United States)

7452 0A A VIIRS ocean data simulator [7452-09]

W. D. Robinson, F. S. Patt, Science Applications International Corp. (United States);

B. A. Franz, NASA Goddard Space Flight Ctr. (United States); K. R. Turpie, Science

Applications International Corp. (United States); C. R. McClain, NASA Goddard Space Flight Ctr. (United States)

\section{SESSION 3 NEW MISSIONS}

7452 OC Desert test site uniformity analysis [7452-11]

D. X. Kerola, Skillstorm Government Integrated Services (United States); C. J. Bruegge, Jet Propulsion Lab. (United States) 
7452 OD SENTINEL-2 image quality and level 1 processing [7452-12]

A. Meygret, Ctr. National d'Études Spatiales (France) and ESA ESTEC (Netherlands); S. Baillarin, Ctr. National d'Études Spatiales (France) and ESA ESRIN (Italy); F. Gascon, ESA ESTEC (Netherlands); E. Hillairet, Magellium (France); C. Dechoz, S. Lacherade, Ctr. National d'Études Spatiales (France); P. Martimort, F. Spoto, ESA ESTEC (Netherlands); P. Henry, Ctr. National d'Études Spatiales (France); R. Duca, ESA ESRIN (Italy)

7452 OE Status of the optical payload and processor development of ESA's Sentinel 3 mission [7452-13]

J. Nieke, J. Frerick, J. Stroede, C. Mavrocordatos, B. Berruti, ESA ESTEC (Netherlands)

7452 OF In-orbit imaging and radiometric performance prediction for flight model Geostationary Ocean Color Imager [7452-14]

S. Jeong, Y. Jeong, D. Ryu, Yonsei Univ. (Korea, Republic of); S. Kim, Korea Aerospace Research Institute (Korea, Republic of); S. Cho, Korea Ocean Research \& Development Institute (Korea, Republic of); J. Hong, I\&A Technology (Korea, Republic of); S.-W. Kim, Yonsei Univ. (Korea, Republic of); H. S. Youn, Korea Aerospace Research Institute (Korea, Republic of)

\section{SESSION 4 EARTH OBSERVING SYSTEM SENSORS I}

7452 OG Improving weather and climate prediction with the AIRS on Aqua [7452-15]

T. S. Pagano, Jet Propulsion Lab. (United States)

$7452 \mathrm{OH} \quad$ Spectral calibration in hyperspectral sounders [7452-16]

E. M. Manning, H. H. Aumann, R. G. Deen, Y. Jiang, Jet Propulsion Lab. (United States);

L. L. Strow, S. E. Hannon, Univ. of Maryland, Baltimore County (United States)

7452 Ol Sensor performance of Clouds and the Earth's Radiant Energy System (CERES) instruments aboard EOS Terra and Aqua spacecraft based on post-launch calibration studies [7452-17] S. Thomas, Science Systems and Applications, Inc. (United States); K. J. Priestley, NASA Langley Research Ctr. (United States); P. C. Hess, R. S. Wilson, Science Systems and Applications, Inc. (United States); M. A. Avery, NASA Langley Research Ctr. (United States); D. R. Walikainen, Z. P. Szewczyk, D. L. Cooper, M. Shankar, Science Systems and Applications, Inc. (United States)

7452 0J On-orbit solar calibrations using the Aqua Clouds and Earth's Radiant Energy System (CERES) in-flight calibration system [7452-18]

R. S. Wilson, Science Systems and Applications, Inc. (United States); K. J. Priestley, NASA Langley Research Ctr. (United States); S. Thomas, P. Hess, Science Systems and Applications, Inc. (United States)

\section{SESSION 5 EARTH OBSERVING SYSTEM SENSORS II}

7452 OK MODIS solar reflective calibration traceability [7452-19]

X. Xiong, J. Butler, NASA Goddard Space Flight Ctr. (United States)

$7452 \mathrm{OL}$ Trends in MODIS geolocation error analysis [7452-20]

R. E. Wolfe, NASA Goddard Space Flight Ctr. (United States); M. Nishihama, NASA Goddard Space Flight Ctr. (United States) and Sigma Space Corp. (United States) 
7452 OM On-orbit operation and performance of MODIS blackbody [7452-21]

X. Xiong, NASA Goddard Space Flight Ctr. (United States); T. Chang, Science Systems and Applications, Inc. (United States); W. Barnes, Univ. of Maryland, Baltimore County (United States)

7452 ON Detector dependency of MODIS polarization sensitivity derived from on-orbit characterization [7452-22]

G. Meister, Futuretech Corp. (United States); B. A. Franz, NASA Goddard Space Flight Ctr. (United States); E. J. Kwiatkowska, ESA ESTEC (Netherlands); R. E. Eplee, SAIC (United States); C. R. McClain, NASA Goddard Space Flight Ctr. (United States)

\section{SESSION 6 SENSORS, TECHNOLOGIES, AND MEASUREMENT TECHNIQUES}

$745200 \quad$ Staggered arrays for high resolution earth observing systems [7452-23]

C. Latry, J.-M. Delvit, Ctr. National d'Études Spatiales (France)

7452 OP Transmittance measurement of a heliostat facility used in the preflight radiometric calibration of Earth-observing sensors [7452-24]

J. Czapla-Myers, College of Optical Sciences, The Univ. of Arizona (United States); K. Thome, NASA Goddard Space Flight Ctr. (United States); N. Anderson, J. McCorkel, N. Leisso, College of Optical Sciences, The Univ. of Arizona (United States); W. Good, S. Collins, Ball Aerospace \& Technologies Corp. (United States)

$74520 Q \quad$ Remote sensing capabilities of the Airborne Compact Atmospheric Mapper [7452-27] M. G. Kowalewski, Science Systems and Applications, Inc. (United States); S. J. Janz, NASA Goddard Space Flight Ctr. (United States)

7452 OR A sampling technique in the star-based monitoring of GOES imager visible-channel responsivities [7452-28]

I-L. Chang, C. Dean, Perot Systems Corp. (United States); M. Weinreb, Riverside Technology, Inc. (United States); X. Wu, National Oceanic and Atmospheric Administration (United States)

\section{SESSION 7 LAND REMOTE SENSING}

7452 OS Landsat-7 and Landsat-5 thermal band calibration updates [7452-29]

J. A. Barsi, B. L. Markham, NASA Goddard Space Flight Ctr. (United States); J. R. Schott, Rochester Institute of Technology (United States); S. J. Hook, Jet Propulsion Lab. (United States); N. G. Raqueno, Rochester Institute of Technology (United States)

7452 OT Performance results for the Landsat OLI spectral filters [7452-30] J. W. Figoski, N. Zaun, Ball Aerospace \& Technologies Corp. (United States); T. Mooney, Barr Associates, Inc. (United States)

7452 OU The increased potential for the Landsat Data Continuity Mission to contribute to case 2 water quality studies [7452-31]

A. Gerace, J. Schott, Rochester Institute of Technology (United States) 
7452 OX The cross calibration of SeaWiFS and MODIS using on-orbit observations of the Moon [7452-35]

R. E. Eplee, Jr., Science Applications International Corp. (United States); X. Xiong, NASA Goddard Space Flight Ctr. (United States); J.-Q. Sun, Science Systems and Applications, Inc. (United States); G. Meister, Futuretech Corp. (United States); C. R. McClain, NASA Goddard Space Flight Ctr. (United States)

7452 OY Characterization of MODIS and SeaWiFS solar diffuser on-orbit degradation [7452-36] X. Xiong, NASA Goddard Space Flight Ctr. (United States); R. E. Eplee, Jr., Science Applications International Corp. (United States); J. Sun, Science Systems and Applications, Inc. (United States); F. S. Patt, Science Applications International Corp. (United States); A. Angal, Science Systems and Applications, Inc. (United States); C. R. McClain, NASA Goddard Space Flight Ctr. (United States)

745210 Radiometric characterization of hyperspectral imagers using multispectral sensors [7452-38] J. McCorkel, College of Optical Sciences, The Univ. of Arizona (United States); K. Thome, NASA Goddard Space Flight Ctr. (United States); N. Leisso, N. Anderson, J. Czapla-Myers, College of Optical Sciences, The Univ. of Arizona (United States)

\section{SESSION 9 EARTH OBSERVATION SYSTEMS AND APPLICATIONS IN CHINA}

$745211 \quad$ Earth observations and their applications in China (Invited Paper) [7452-46]

X. Gu, Institute of Remote Sensing Applications (China); B. Song, Defense Industry Program Evaluation Ctr. of SASTIND (China); J. Wang, X. Zhou, Institute of Remote Sensing Applications (China)

745213 The microwave sensor status and future developing plan of China meteorological satellites [7452-48]

H. Yang, N. Lu, Z. Ge, H. Yin, China Meteorological Administration (China)

\section{POSTER SESSION}

745216 An enhanced vegetation cover method for automatic generation of land surface emissivity maps [7452-39]

E. Caselles, Univ. Politécnica de València (Spain) and Univ. de València (Spain); F. J. Abad, Univ. Politécnica de València (Spain); E. Valor, J. M. Galve, V. Caselles, Univ. de València (Spain)

745217 Characterization of MODIS SD screen vignetting function using observations from spacecraft yaw maneuvers [7452-40]

Z. Wang, Science Systems and Applications, Inc. (United States); X. Xiong, NASA Goddard Space Flight Ctr. (United States)

745218 MODIS solar diffuser stability monitor: function and applications [7452-41]

H. Chen, Science Systems and Applications, Inc. (United States); X. Xiong, NASA Goddard Space Flight Ctr. (United States) 
745219 Time-dependent response versus scan angle for MODIS reflective solar bands [7452-43] J. Sun, Science Systems and Applications, Inc. (United States); X. Xiong, NASA Goddard Space Flight Ctr. (United States); H. Chen, A. Angal, Science Systems and Applications, Inc. (United States); X. Geng, SAIC (United States); A. Wu, Science Systems and Applications, Inc. (United States)

$74521 \mathrm{~A} \quad$ Long-term monitoring of radiometer sensitivity for radiometric comparisons among optical laboratories [7452-44]

G. Meister, Futuretech Corp. (United States); G. S. Fargion, San Diego State Univ. (United States); C. R. McClain, NASA Goddard Space Flight Ctr. (United States)

Author Index 
Downloaded From: https://www.spiedigitallibrary.org/conference-proceedings-of-spie on 26 Apr 2023

Terms of Use: https://www.spiedigitallibrary.org/terms-of-use 


\title{
Conference Committee
}

\author{
Program Track Chair
}

Allen H.-L. Huang, University of Wisconsin, Madison (United States)

Conference Chairs

James J. Butler, NASA Goddard Space Flight Center (United States)

Xiaoxiong Xiong, NASA Goddard Space Flight Center (United States)

Cochair

Xingfa Gu, Institute of Remote Sensing Applications (China)

Program Committee

Philip E. Ardanuy, Raytheon Intelligence \& Information Systems (United States)

Robert A. Barnes, NASA Goddard Space Flight Center (United States) and Science Applications International Corporation (United States)

Jeffrey S. Czapla-Myers, College of Optical Sciences, The University of Arizona (United States)

Armin W. Doerry, Sandia National Laboratories (United States)

Thomas S. Pagano, Jet Propulsion Laboratory (United States)

Carl F. Schueler, Orbital Sciences Corporation (United States)

Session Chairs

1 Prelaunch Calibration

Xiaoxiong Xiong, NASA Goddard Space Flight Center (United States)

2 NPOESS Preparatory Project

Kurtis J. Thome, NASA Goddard Space Flight Center (United States)

3 New Missions

Armin W. Doerry, Sandia National Laboratories (United States)

$4 \quad$ Earth Observing System Sensors I

Robert A. Barnes, NASA Goddard Space Flight Center (United States) and Science Applications International Corporation (United States) 
5 Earth Observing System Sensors II

Robert A. Barnes, NASA Goddard Space Flight Center (United States) and Science Applications International Corporation (United States)

6 Sensors, Technologies, and Measurement Techniques

Denis A. Elliott, Jet Propulsion Laboratory (United States)

$7 \quad$ Land Remote Sensing

Jeffrey S. Czapla-Myers, College of Optical Sciences, The University of Arizona (United States)

8 Cross-calibration

Philip E. Ardanuy, Raytheon Intelligence \& Information Systems (United States)

9 Earth Observation Systems and Applications in China Xingfa Gu, Institute of Remote Sensing Applications (China) 